\section{1) Das medulläre Karzinom der Niere}

Das medulläre Karzinom der Niere ist ein seltener, aggressiver Tumor, der 1995 erstmals am Armed Forces Institute of Pathology, Washington, DC, als eigenständige Tumorentität definiert wurde (Davis et al., The American Journal of Surgical Pathology 1995; 19(1):1). Der Tumor ist bisher mit einer Ausnahme bei jungen schwarzen Patienten mit Sichelzellanämie diagnostiziert worden. Im Folgenden berichten wir über einen weiteren Fall eines medullären Nierenkarzinoms bei einer weißen Patientin ohne Anhalt für eine Sichelzellanämie.

\section{Fallbeschreibung}

Im August 1999 erfolgte die stationäre Aufnahme einer 44jährigen Kaukasierin wegen massiver Schmerzen im Bereich des linken Hüftgelenkes und proximalen Oberschenkels nach einem Sturztrauma.

Röntgenologisch zeigte sich eine Osteodestruktion im Bereich des linken proximalen Femurs mit pathologischer Fraktur, die mittels einer dynamischen Hüftschraube stabilisiert wurde.

Das während der Operation entnommene Material aus dem Frakturbereich ergab histologisch Anteile einer Knochenmetastase eines mäßig gut differenzierten Adenokarzinoms. Als Lokalisation für den Primärtumor wurden Mamma oder Lunge angegeben, differentialdiagnostisch kam von Seiten des Pathologen auch der Verdauungstrakt (z.B. Pankreas, Gallenwege, etc.) in Betracht. Für ein klassisches Nierenzellkarzinom fanden sich anhand des Materials keine Hinweise, ebenso wenig für ein Urothelkarzinom (Institut für Pathologie, Klinikum Nürnberg, H 27619/99).

Daraufhin erfolgte ein umfangreiches Tumorstaging. Bei unauffälligem Tastbefund ergab sich auch sonographisch und mammographisch kein Anhalt für ein Mammakarzinom. Die Thoraxübersichtsaufnahme zeigte eine Verdichtungsstruktur im Bereich des linken Hilus, die übrigen Lungenparenchymstrukturen waren regelrecht. Im Thorax-CT ließ sich eine noduläre Veränderung im Bereich des linken Hilus nachweisen, die am ehesten einer hilären Lymphadenopathie entsprach. Die übrigen Thoraxstrukturen kamen unauf- fällig zur Abbildung. Die anschließend durchgeführte Bronchoskopie ergab keinen Hinweis auf einen Bronchialtumor.

Im Abdomen-CT fand sich im mittleren Parenchymdrittel der linken Niere ein $4 \mathrm{~cm}$ großer, expansiv wachsender, nativ inhomogener Tumor, der zu einer Vorwölbung der lateralen Parenchymkontur führte und bis an die Faszia Gerota heranreichte. Nach Kontrastmittelgabe demarkierte sich ein überwiegend nekrotischer, partiell unscharf begrenzter Tumor mit randständig soliden Anteilen und peripherem Enhancement. Eine Infiltration des Nierenhohlsystems war nicht nachweisbar. Lokoregionär und paraaortal fanden sich keine vergrößerten Lymphknoten. Darüber hinaus stellte sich eine $5 \mathrm{~cm}$ große Osteodestruktion mit Weichteilkomponente im Bereich des linken Osilium dar (Abb. 1 a und b). Im i.v. Urogramm kam ein normalweites, regelrecht konfiguriertes Nierenbeckenkelchsystem links ohne Hinweis auf eine Tumorinfiltration oder eine Abflussstörung zur Abbildung. Die laterale Parenchymkontur im mittleren Drittel der linken Niere war nicht abgrenzbar (Abb. 2).

Ein F-18-FDG-Ganzkörper-PET zeigte multiple Foki mit vermehrter Tracerspeicherung links supraclavikulär, im Bereich des linken Hilus, des bekannten Nierentumors links, links parailiakal, der linken Beckenschaufel, des rechten Schenkelhalses und der inter- und subtiochantären Region des linken proximalen Femurs (Abb. 3).

Trotz der Diskrepanz zwischen histologischem Befund und radiologischer Verdachtsdiagnose eines metastasierten Nierentumors erfolgte im September 1999 die Tumornephrektomie.

Die histologische Aufarbeitung des Resektates ergab ein 5,5 cm großes, wenig differenziertes Nierenkarzinom, im Speziellen ein renales medulläres Karzinom, mit Infiltration in das pararenale Fettgewebe ohne Durchbruch der Fascia Gerota mit Lymph- und Hämangiosis carzinomatosa. Zusätzlich waren mehrere kleine, um den Haupttumor gruppierte Satellitentumoren nachweisbar. In Zusammenschau mit den Vorbefunden wurde retrospektiv die bekannte
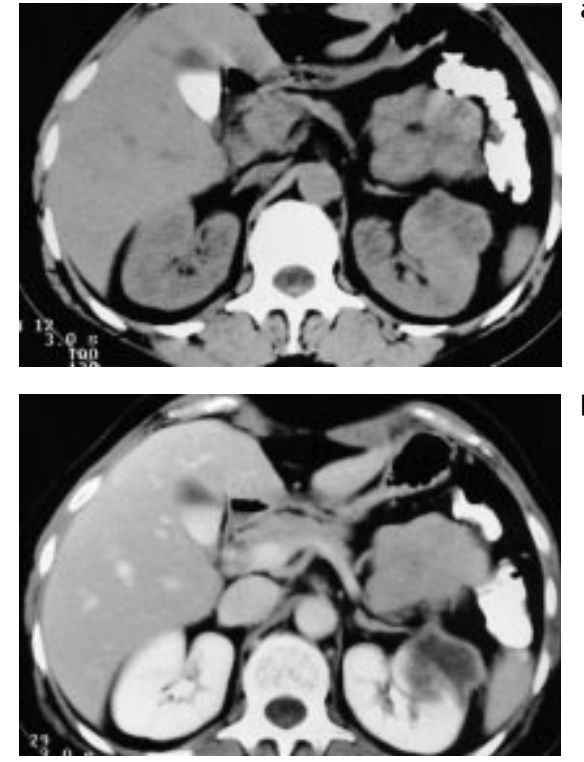

Abb.1 Abdomen-CT. (a) Nativ inhomogen strukturierter, expansiv wachsender Tumor im mittleren Parenchymdrittel der linken Niere mit Vorwölbung der Nierenkontur. (b) Nach Kontrastmittelgabe überwiegend hypovaskularisierter, partiell unscharf begrenzter Tumor mit peripherem Enhancement. Ausdehnung bis zur Faszia Gerota, keine Infiltration des Nierenhohlsystems.

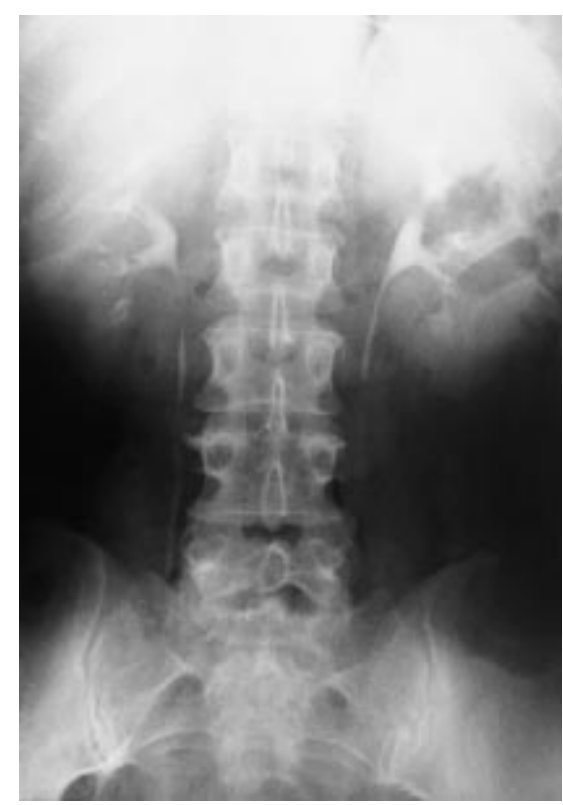

Abb. 2 i.v. Urogramm. Unauffällige Darstellung des Nierenbeckenkelchsystems beidseits ohne Abflussstörung. Laterale Kontur der linken Niere nicht abgrenzbar.

ossäre Metastase im Bereich des linken proximalen Femurs als Absiedelung dieses Tumors eingestuft (Tumorklassifikation G3 , pT3a, L1,V1) (H33434/99). 


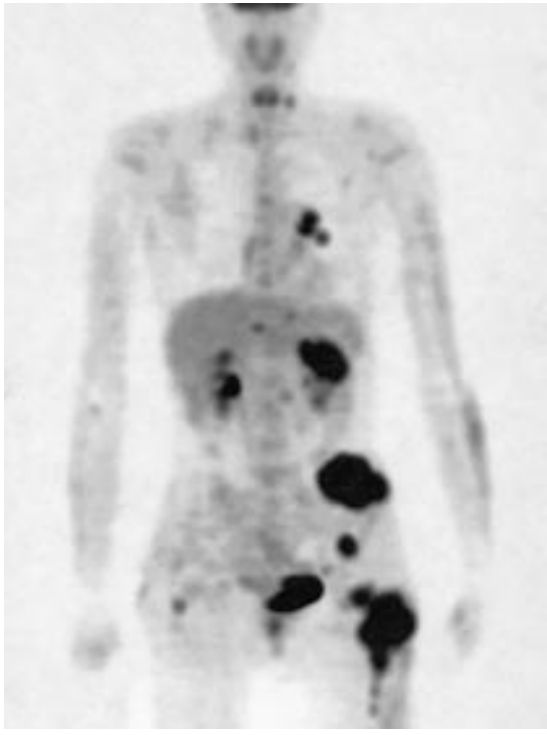

Abb. 3 F-18-FDG-Ganzkörper-PET. Multiple Mehrspeicherungen links supraclaviculär, im Bereich des linken Hilus, der linken Niere, links parailiakal, der linken Beckenschaufel, des rechten Schenkelhalses sowie der inter- und subtrochantären Region des linken proximalen Femurs.

Postoperativ wurden bei der Patientin inzwischen mehrfach palliative Strahlentherapien verschiedener Lokalisationen im Skelettsystem durchgeführt.

\section{Diskussion}

Das medulläre Nierenkarzinom ist ein seltener, aggressiver Tumor, der bislang mit einer Ausnahme bei jüngeren schwarzen Patienten im Alter zwischen 11 und 39 Jahren mit Sichelzellanämie beschrieben wurde (Herring et al.; The Journal of Urology 1997; 157: 2246).

Das biologische Verhalten des Tumors ist durch ein lokal infiltratives Wachstum und eine frühe Metastasierung in Lymphknoten, Leber und Lunge charakterisiert (Avery et al., Cancer 1996; 78: 128). Die von Davis et al. analysierten 33 Patienten hatten bereits zum Zeitpunkt der Diagnosestellung extrarenale Tumormanifestationen bei einer mittleren Überlebenszeit von drei Monaten. Therapieversuche mit verschiedenen Chemotherapieschemata konnten bislang keine Verbesserung der infausten Prognose herbeiführen.

Als wahrscheinlicher Entstehungsort des Tumors wird das Epithel innerhalb oder in Nachbarschaft einer Nierenpapille diskutiert.
Das makroskopische Bild entspricht einem unscharf begrenzten, das Nierenparenchym infiltrierenden Tumor mit Hämorrhagien und Nekrosen. Bei der Mehrzahl der publizierten Fälle wurden eine Tumorausdehnung in das Nierenbeckenkelchsystem mit Ektasie sowie kleine Satellitenknoten in der Nierenrinde beschrieben. Der durchschnittliche Tumordurchmesser betrug ca. $6 \mathrm{~cm}$.

Histologisch imponiert eine dottersackähnliche Morphologie mit adenoid-zystischen Veränderungen. Bei ausgeprägter desmoplastischer Stromareaktion finden sich meist gering differenzierte Tumorzellen mit großen, hellen Kernen. Daneben werden in variabler Ausprägung polymorphkernige Leukozyten im Tumorkern und Lymphozytensäume an den Tumorrändern, auch der Satellitenknoten, vorgefunden.

Die bildgebenden Verfahren zeigen typischerweise einen zentral infiltrativ wachsenden, überwiegend nekrotischen, tumorösen Prozess mit Ausdehnung in den Nierensinus und die -rinde. Nach KM-Applikation kommt es zu einem peripheren Enhancement mit zentraler Hypodensität. Im fortgeschrittenen Stadium überschreitet der Tumor die Organgrenzen, wobei die Niere ihre ursprüngliche Form beibehält. Differentialdiagnostisch ist das medulläre Nierenkarzinom vom häufigeren Nieren-zellkarzinom, das charakteristischerweise in der Nierenrinde lokalisiert ist und eine expansive (vs. infiltrative) Wachstumsform aufweist, vom Urothelkarzinom im Bereich des Nierenbecken- kelchsystems, vom Lymphom und von Metastasen z. B. eines Plattenepithelkarzinoms, abzugrenzen (Davidson AJ, Hartmann DS (eds.). Radiology of the kidney and urinary tract. Philadelphia: Saunders 1994).

In unserem Falle wurde das medulläre Nierenkarzinom nicht in die Differentialdiagnose mit einbezogen, da die bildmorphologischen Befunde wie expansives Tumorwachstum und ossäre Metastasierung auf ein primäres Nierenzellkarzinom hinwiesen.

Die F-18-FDG-Ganzkörper-PET ist aufgrund des gesteigerten Glukosemetabolismus ein sensitives Verfahren für den Nachweis von malignen Tumoren und deren Metastasen. In unserem Fall wird zum ersten Mal über den Einsatz der F18-FDG-PET beim medullären Nierenkarzinom berichtet und die in der Literatur beschriebene, frühe lymphogene und hämatogene Metastasierung des Tumors bestätigt.

Nach unseren Literaturrecherchen handelt es sich hier um den 2. Fall eines medullären Nierenkarzinoms bei einem nicht negroiden Patienten ohne Hinweis auf eine Sichelzellanämie. In Übereinstimmung mit Kalyanpur et al. unterstützt dies die Hypothese, dass das medulläre Nierenkarzinom sowohl als genetisch assoziierte als auch sporadische Verlaufsform vorkommen kann (Kalyanpur et al.; AJR 1997, 169: 1037).

\section{B. Glas, F. J. Ferstl, Nürnberg}

